\title{
Unroofed Midline Prostate Cyst Misled Into a Stricture With Obliterative Bladder Neck Contracture Following a Laser Prostatectomy
}

\author{
Richilda Red Diaz ${ }^{1}$ Joo Yong Lee ${ }^{1}$, Young Deuk Choi ${ }^{1,2,3}$, Kang Su Cho \\ ${ }^{1}$ Department of Urology, Urological Science Institute, ${ }^{2}$ Clinical Trial Center for Medical Device, ${ }^{3}$ Robot and Minimal Invasive Surgery Center, Severance \\ Hospital, Yonsei University College of Medicine, Seoul, Korea
}

\begin{abstract}
We report on a case of a 67-year-old man who presented with persistent lower urinary tract symptoms following a potassium titanyl phosphate laser photoselective vaporization of prostate. Upon further diagnostic examinations were performed, he was noted to have an obliterative bladder neck contracture with an incidental, misleading, and rare presence of an unroofed midline anterior prostatic cyst presenting as a stricture. As we were presented with this case, it was imperative to address these complications of bladder neck contracture and incompletely ablated prostatic cyst. This report brings to light underestimated complicating factors in the urinary tract, and the diagnostic and therapeutic interventions we had undertaken to rectify the identified complications and improve patient's quality of life. The patient underwent internal urethrotomy, resection of prostatic cyst wall and transurethral resection of the prostate directed to improve his quality of life and prevent urinary retention.
\end{abstract}

Keywords: Urinary bladder neck obstruction; Urethra; Prostate; Cysts

Bladder neck contracture/stenosis or strictures although rare, are conditions that can strongly impair the patient's quality of life (QoL) especially when it is a complication for a treatment, which the patient has sought for. The majority of patients with stricture experience moderate complications, such as bother from lower urinary tract voiding symptoms, recurrent urinary tract infection and the need for repeat urethral procedures such as dilation or urethrotomy [1]. In industrialized countries, strictures more commonly derive from iatrogenic instrumentation, external trauma, and, to a much lesser degree, inflammatory diseases like lichen sclerosus et atrophicus [2]. We report an unusual case of obliterative bladder neck contracture with an incidental, misleading, and rare presence of an unroofed midline anterior prostatic cyst following a potassium titanyl phosphate (KTP) laser to perform photoselective vaporization of prostate (PVP). To our knowledge, this is the first case report of a prostatic cyst appearing as a pseudo-bladder neck contracture and at the same time masking a true bladder neck contracture.

\section{CASE REPORT}

A 67-year-old man visited Severance Hospital due to persistent and aggravated lower urinary tract symptoms (LUTS) following a KTP laser PVP 3-month ago in another hospital. The International Prostate Symptom Score (IPSS) was 21, the QoL score was 5 . Uroflowmetry showed that the peak flow rate was $1.8 \mathrm{~mL} / \mathrm{sec}$. Transrectal ultrasonography revealed prostate volume was approximately $35 \mathrm{~mL}$ (Fig. 1A). A bladder neck contracture was considered, and transurethral incisions of the bladder neck and possible completion transurethral resection of the prostate (TURP) were planned.

Under the spinal anesthesia, patient was placed in the lithot-
Corresponding author: Kang Su Cho

Department of Urology, Urological Science Institute, Severance Hospital, Yonsei University College of Medicine, 50 Yonsei-ro, Seodaemun-gu, Seoul 120-752, Korea

Tel: +82-2-2228-2320 / Fax: +82-2-312-2538 / E-mail: kscho99@yuhs.ac Submitted: June 15, 2012 / Accepted after revision: March 6, 2013
This is an Open Access article distributed under the terms of the Creative Commons Attribution Non-Commercial License (http://creativecommons.org/licenses/by-nc/3.0/) which permits unrestricted non-commercial use, distribution, and reproduction in any medium, provided the original work is properly cited. 
omy position. Using a 24 Fr resectoscope (Karl Storz, Tuttlingen, Germany), cystoscopy revealed an elevated bladder neck with narrow opening. Transurethral resection at the narrow vesical neck was performed. On panendoscopy, vascular pattern with areas of prominent vessels of the bladder mucosa appeared unremarkable. However, other anatomic landmarks such as the trigone and ureteral orifices could not be identified despite giving intravenous indigo-carmine, and the bladder capacity was noted to be extremely small. So we suspected that this space, which was regarded as the bladder, might not be a true vesical space. A palpable suprapubic mass was noted on Crede maneuver, which was identified as a distended bladder on an intraop-
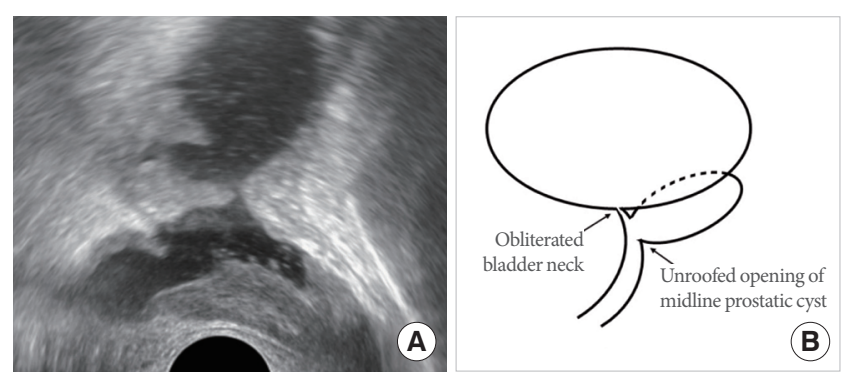

Fig. 1. (A) Transrectal ultrasonography showed that prostate may be resected by previous photoselective vaporization of prostate. (B) Schematic illustration on the relationship between obliterated bladder neck and midline prostatic cyst.
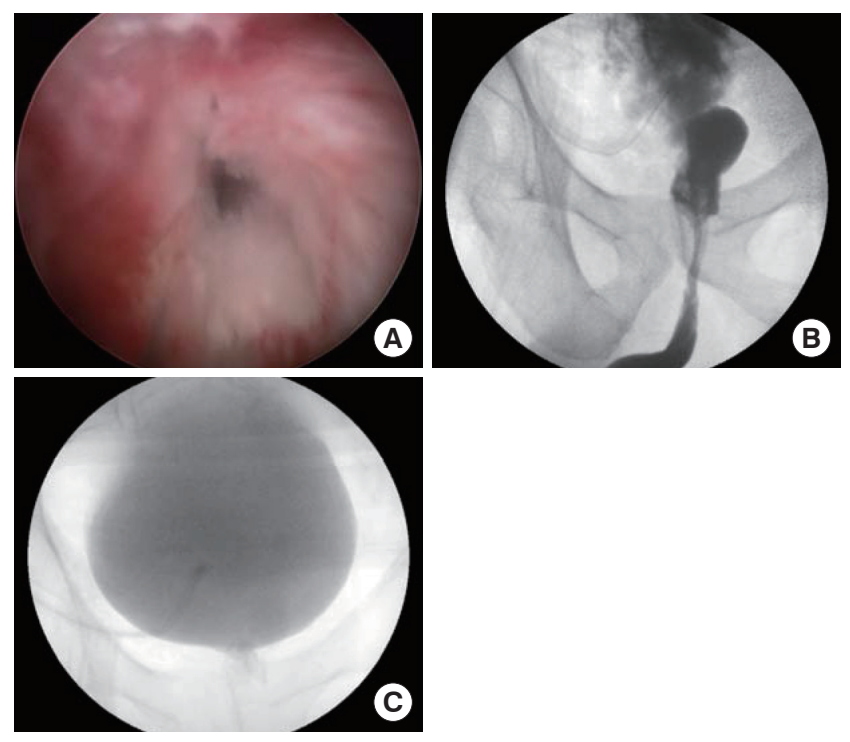

Fig. 2. (A) The true bladder neck contracture; (B) Urethrography revealed the anatomy of the anterior urethra and an outline of midline prostatic cyst of considerable size. (C) Cystography that shows a distended bladder. erative ultrasonography. A suprapubic cystostomy was performed to adequately drain the bladder and thus address the obstruction temporarily. Retrograde urethrography revealed severe bladder neck obstruction and a large midline cyst (Fig. 2). In retrospect, what we visualized by initial cystoscopy and assumed as the bladder neck, were actually the borders of an unroofed cyst from the previous PVP. Furthermore, the cyst wall seeming to appear like a bladder mucosa was what we assumed to be as the urinary bladder (Fig. 3). Under these thorough anatomical understanding, the severely obliterated bladder contracture ("true bladder opening") was identified through a repeat endoscopic evaluation. A straight urethrotome was advanced to create a channel at bladder neck, and more radical incisions made from the minute opening at the site of the contracture to create a larger opening. The area of the contracture was further resected to achieve visualization of the bladder and its entirety. This time the bladder trigone and ureteral orifices were identified, and no other abnormalities were seen. Resection of the prostatic cyst wall was done extending to the fibromuscular area of the prostate gland. A three-way Foley catheter was inserted after evacuation of the resected tissues. The patient was discharged with both Foley catheter and suprapubic catheter maintained. A week after the procedure, the patient's Foley catheter was removed and he was noted to have resolution of
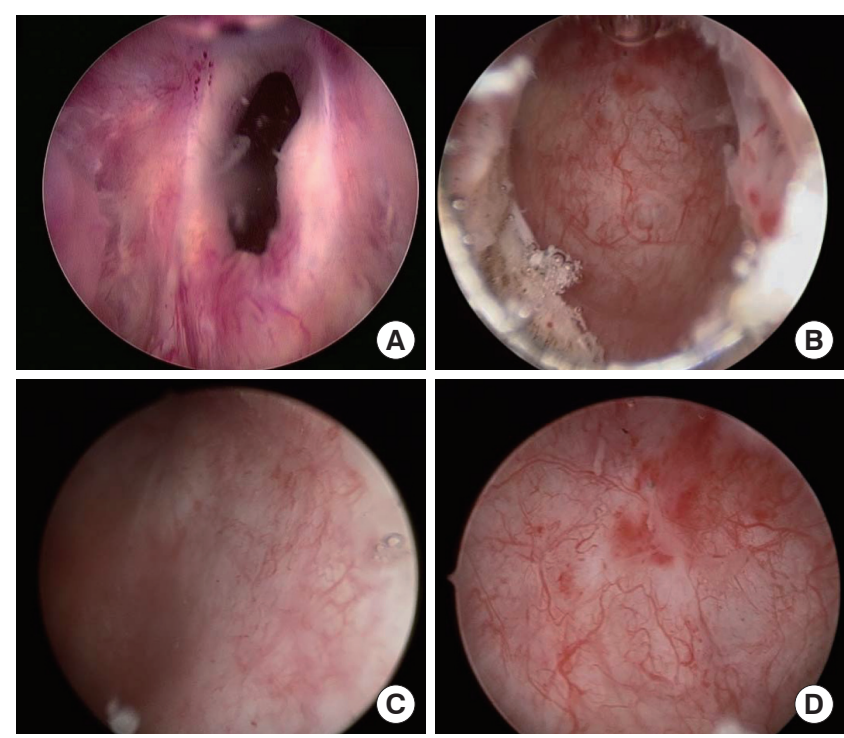

Fig. 3. (A) A misleading appearance of the borders of an unroofed cyst wall assumed to be an elevated and contracted bladder neck. (B) The resected borders of the misleading cyst; (C, D) the mucosal vascular pattern of the cyst wall appearing like a bladder mucosa. 

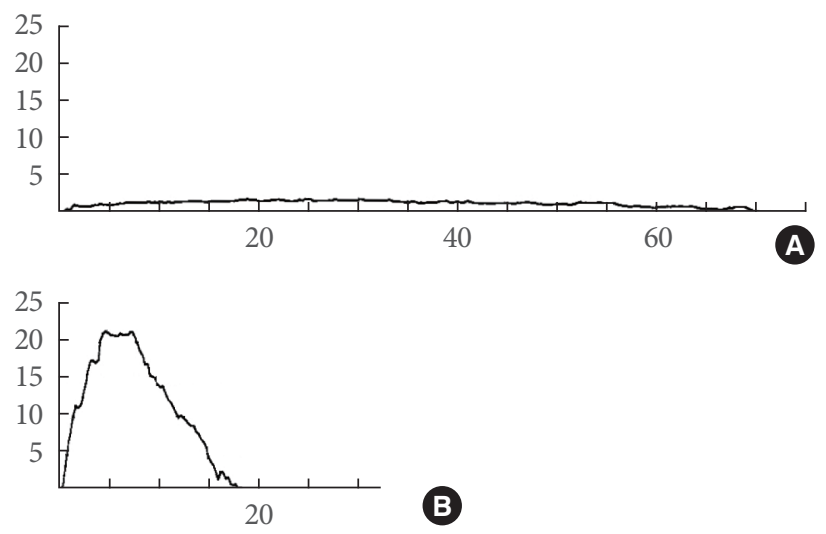

Fig. 4. (A) The preoperative uroflowmetry pattern suggested obstructive flow (maximal flow rate [Qmax], $1.3 \mathrm{~mL} / \mathrm{sec}$ ). (B) In postoperative uroflowmetry, patient's symptom was improved $(\mathrm{Q} \max =21.5 \mathrm{~mL} / \mathrm{sec})$.

voiding symptoms. Removal of the clamped suprapubic catheter was done later when voiding problems were noted to be resolved since it can be used to drain the bladder should the patient have difficulty in voiding. A noninvasive procedure, uroflowmetry was repeated on follow up and showed a flow rate of $21.5 \mathrm{~mL} / \mathrm{sec}$ and a residual volume of $8 \mathrm{~mL}$ (Fig. 4). IPSS was 7 and QoL was 2, 3 months after operation.

\section{DISCUSSION}

So far, in published reports, the total number of cases of symptomatic midline prostatic cysts located anteriorly is fewer than five [3]. Prostatic cysts are observed in $0.5 \%$ to $7.9 \%$ of patients and are classified into six distinct types including: 1) isolated medial cysts, 2) cysts of the ejaculatory duct, 3) simple or multiple cysts of the parenchyma, 4) complicated cysts (infectious or hemorrhagic), 5) cystic tumors, and 6) cysts secondary to parasitic disease [4]. The cyst may appear to obstruct the bladder outlet by a ball-valve mechanism as previously reported $[5,6]$. Some cystic lesions in the male pelvis may be discovered by chance with sonography that is now more widely used; in other cases, however, they may remain overlooked [7]. Associated symptoms include irritation and/or obstructing voiding symptoms, decreased volume of ejaculate, painful ejaculation, hematospermia, and infertility [5]. In a sonographic-pathologic correlation done by Hamper et al. [8], cystic lesions were not associated with carcinoma of the prostate, but represented either a growth phenomenon related the presence of benign prostatic hypertrophy, inflammatory conditions (abscesses), or anatomic variants (utricle). Incompletely excised cysts during open surgery are reported to recur, whereas durable, recurrence-free results have been reported for medial prostatic cysts treated with transurethral approach [9].

From these literature gathered, prostatic cyst is a relatively rare entity, it can be congenital or acquired, an incidental finding on diagnostic examinations such as ultrasonography, can be overlooked, and can lead to misdiagnosis when it becomes symptomatic. In review of the events, this patient presented with LUTS that prompted him to seek medical advice. A prostatic cyst as an isolated cause of bladder outlet obstruction may have been treated with a transurethral resection of the prostatic cyst and TURP and might prevented a bladder neck contracture. As we were presented with this rare case, it was imperative to address these complications of bladder neck contracture and incompletely ablated prostatic cyst. It was amazing for us do discover that an unroofed prostatic cyst can appear like bladder neck contracture, and its wall appear like a bladder mucosa on cystoscopy (Fig. 1B). Although the ball-valve mechanism of prostatic cysts has been reported in the past, no report on a cyst wall appearing like a bladder mucosa on cystoscopy has been reported. The urethrogram performed also revealed the outline of a prostatic cyst with considerable size.

Apparently in this case, the patient presented with severe LUTS and was aggravated even after PVP. It was an aim to relieve the obstruction, provide definitive treatment and improve QoL. A suprapubic tube cystostomy as a short-term treatment option was performed. An internal urethrotomy and resection of the true bladder neck contracture that was most likely iatrogenic in nature, was performed to create a tunnel and promote re-epithelization before scarring contacts. Transurethral resection of a dense scar at the bladder neck may help relieve the obstruction but care must be taken to avoid aggressive resection or risk fistula formation [10]. A resection of the bladder neck contracture was performed as well as transurethral resection of cyst wall and completion TURP.

In conclusion, urologist should pay attention to patients who show abnormal findings following laser prostatectomy or TURP, and consider possibility of unusual structural abnormalities similar with our case.

\section{CONFLICT OF INTEREST}

No potential conflict of interest relevant to this article was reported. 


\section{REFERENCES}

1. Santucci RA, Joyce GF, Wise M. Male urethral stricture disease. J Urol 2007;177:1667-74.

2. Ferguson GG, Bullock TL, Anderson RE, Blalock RE, Brandes SB. Minimally invasive methods for bulbar urethral strictures: a survey of members of the American Urological Association. Urology 2011; 78:701-6.

3. Lee JY, Kang DH, Park HY, Park JS, Son YW, Moon HS, et al. An anteriorly positioned midline prostatic cyst resulting in lower urinary tract symptoms. Int Neurourol J 2010;14:125-9.

4. Galosi AB, Montironi R, Fabiani A, Lacetera V, Galle G, Muzzonigro G. Cystic lesions of the prostate gland: an ultrasound classification with pathological correlation. J Urol 2009;181:647-57.

5. Issa MM, Kalish J, Petros JA. Clinical features and management of anterior intraurethral prostatic cyst. Urology 1999;54:923.

6. Dell'Atti L. Disorders of bladder depletion in a young man because of a prostatic cyst: a case report. Arch Ital Urol Androl 2012;84:44-6. 7. Ishikawa M, Okabe H, Oya T, Hirano M, Tanaka M, Ono M, et al. Midline prostatic cysts in healthy men: incidence and transabdominal sonographic findings. AJR Am J Roentgenol 2003;181:1669-72.

8. Hamper UM, Epstein JI, Sheth S, Walsh PC, Sanders RC. Cystic lesions of the prostate gland. A sonographic: pathologic correlation. J Ultrasound Med 1990;9:395-402.

9. Nayyar R, Dogra PN. Anteriorly placed midline intraprostatic cyst. J Endourol 2009;23:595-7.

10. Breyer BN, McAninch JW. Management of recalcitrant bladder neck contracture after radical prostatectomy for prostate cancer. Endoscopic and open surgery. J Urol 2011;185:390-1. 